\title{
CC MATE Tablets: A Well Balanced Calcium Supplement for Well-Functioning of Intestinal Ecosystem \& for Healthy Body
}

Govind Shukla, C. Subrahmanyam, Mantipally Yamuna \& C.J. Sampath Kumar

Lactonova Nutrition Research Centre, Hyderabad.

A Unit of Lactonova Nutripharm (P) Ltd., 81/3, IDA Mallapur, Hyderabad, Telangana-500076, India.

DOI: http://doi.org/10.46382/MJBAS.2021.5407

Copyright: $\odot 2021$ Govind Shukla et al. This is an open access article distributed under the terms of the Creative Commons Attribution License, which permits unrestricted use, distribution, and reproduction in any medium, provided the original author and source are credited.

Calcium is very essential in muscle contraction, oocyte activation, building strong bones and teeth, blood clotting, nerve impulse, transmission, regulating heart beat and fluid balance within cells. The requirements are greatest during the period of growth such as childhood, during pregnancy, when breast feeding. Long term of calcium deficiency can lead to oestoporosis in which the bone deteriorates and there is an increased risk of fractures. Eating a well-balanced calcium supplement can provide all the necessary nutrients and help prevent calcium deficiency. The present paper Reviews the Role of CC Mate tablets developed by Research \& Development cell of Lactonova Nutripharm Pvt Ltd., Hyderabad in maintaining optimum health and wellbeing.

Keywords: Calcium, Oesteoporosis, Hypo and Hypercalcaemia, Parathyroid glands, CC Mate tablets.

\section{Introduction}

Calcium is a mineral that is essential for many aspects of health, including the health of bones and teeth, and a normal heart rhythm. This mineral is also required for muscle contractions and relaxation, nerve and hormone function, and blood pressure regulation.CC Mate tablets can provide all the necessary nutrients and helps to prevent calcium deficiency. Calcium citrate malate in CC Mate tablets is calcium bound citrate and malic acid clinically proven as one of the most absorbable calcium form with the solubility as high as $42 \%$ compared to $22 \%$ of calcium carbonate. Fructoligosaccharides in CC Mate tablets is Prebiotic for well-functioning of intestinal ecosystem and for healthy body. It also boosts absorption of calcium and Magnesium. Vitamin D2 in CC Mate tablets Increases bone mass and reduces the risk of fracture. Magnesium in CC Mate tablets is Amino acid chelated mineral. Zinc, copper and Manganese in CC Mate tablets plays vital support for bone metabolism.

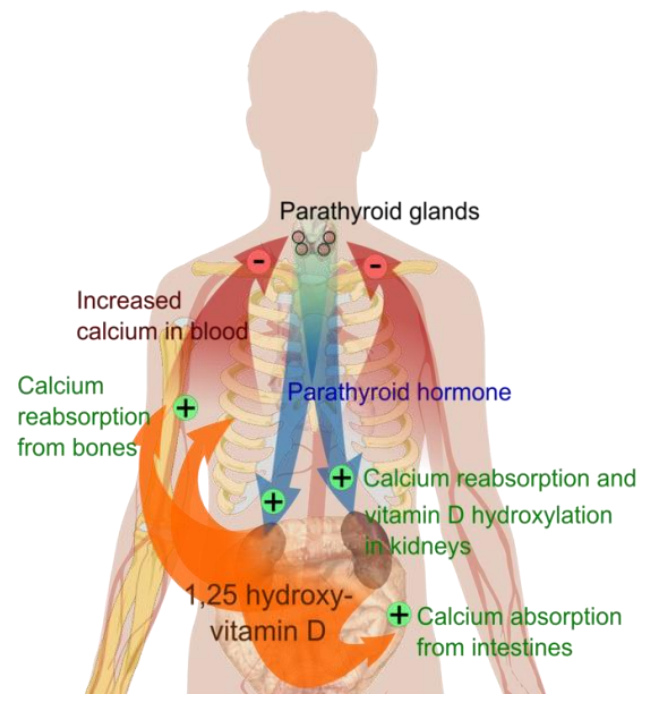

Fig.1. Calcium Regulation (Source: https://med.libretexts.org) 


\section{Physiological role of calcium}

Daily body requirement is about $450 \mathrm{mg}$. The adult requirements of Ca vary. During pregnancy and lactation, there is greater depletion of $\mathrm{Ca}$ from the mother and the intake needs to be increased. On an average, $10 \mathrm{mg}$ per $\mathrm{Kg}$ of body weight per day should be sufficient. Growing children would require from 40 to $60 \mathrm{mg}$ per day. A Cow's milk contains $0.126 \%$ of $\mathrm{Ca}$. A litre of Cow's milk provides therefore a full day's ration of Ca is readily assimilable form. Generally sufficient $\mathrm{Ca}$ gets ingested through the normal through the normal diet. It gets from upper intestinal tract and is excrete through urine and faeces. As the upper portion of intestine the condition is acidic, it tends to favour absorption of $\mathrm{Ca}$. As Ca salts have better solubility.

The alkaline condition brings about the precipitation of Ca salts and the absorption is retarded. Higher fatty acid contents also decreases the absorption due to formation of Ca salts of fatty acid which are insoluble. Calcium is essential to maintaining total body health. Our body needs it every day not just to keep our bones and teeth strong over our life time but to ensure proper functioning of muscles and nerves. It even helps our blood clot. Many peoples think they are getting enough Ca every day but the fact is, they are not so. Ca deficiency is usually due to an inadequate intake of $\mathrm{Ca}$ when blood $\mathrm{Ca}$ levels drop too low, the vital mineral is borrowed from the bones. It is returned to the bones from $\mathrm{Ca}$ supplied through the diet. If an individual's diet is low in $\mathrm{Ca}$, there may not be sufficient amount of $\mathrm{Ca}$ available in the blood to be returned to the bones to maintain strong bones and total body health. Taking Ca regularly every day is a key for preventing and treating Ca deficiency.

According to the U.K. Dept. of Health recommended reference nutrients intake for Ca required according to age. The infants and children require 350-550 mg/day. Teenage girls and boys: 800-1000mg/day, Adult men and women: $700 \mathrm{mg} / \mathrm{day}$. The Ca plays an important role to maintain some important body functions such as:

(a) Ca controls nerve excitability. The effect is mainly on the peripheral neuromuscular mechanism. Fibrillary twitching can be produced by per fusing a muscle with $\mathrm{Ca}$ free fluid. Automatic ganglia also become hyperirritable.

(b) It is necessary for the maintenance of the integrity of the skeletal muscles. An increase in the ionized Ca results in an increase in contractility and vice versa.

(c) It is very vital for maintaining the tone and contractility of heart. $\mathrm{Ca}$ is antidotal to the depressant action of $\mathrm{K}$.

(d) It Aids rennin in the coagulation of milk in the stomach.

(e) It is essential for the clotting of food. It decreases cellular permeability. It is therefore used in allergic conditions to diminish exudation which produces wheals and rushes. Ca appears to serve as a constituent of the intercellular cement.

(f) Ca takes part in the formation of certain tissue and bones. Normally $25-35 \%$ is excreted in the urine and the rest in the stools.

A high protein diet especially derived from animal foods causes $\mathrm{Ca}$ loss in the body. The higher sulphur to calcium ratio of metal increases $\mathrm{Ca}$ excretion and a diet rich in meal can cause bone demineralization. A report published in 
1988comparing the amounts of Ca excreted in the urine showed that, the animal - protein diet cause greater loss of bone loss and hence Oestoporosis. It is the major cause of bone fractures in the elderly. It is better prevented than treated and prevention includes an adequate intake of $\mathrm{Ca}$ throughout life but especially in childhood and young adulthood and minimizing risk factors e.g., smoking, heavy alcohol use and lack of physical exercise. Diet high in protein and in salt also increase Ca loss from the body and may have an effect on oestoporosis. Postmenopausal women are more prone to osteoporosis because they produce less oestrogen which protects the skeleton in younger women.

\section{Calcium Deficiency}

Calcium deficiency is a condition in which the body has an inadequate amount of calcium. Calcium is a mineral that is essential for many aspects of health, including the health of bones and teeth, and a normal heart rhythm. This mineral is also required for muscle contractions and relaxation, nerve and hormone function, and blood pressure regulation. Calcium must be ingested daily and absorbed effectively in order to maintain optimal health. Most people can get enough calcium by eating a variety of foods rich in calcium. Foods that naturally contain calcium include milk and other dairy products; green, leafy vegetables; seafood, nuts, and dried beans. Calcium is also added to orange juice, breakfast cereals, breads, and other fortified food products.

High dietary calcium intake is necessary for infants, children and adolescents in order to promote bone growth and formation. Pregnant women also have higher calcium needs, because it is required for the normal development of fetal bones. In addition, women who have reached menopause need to ensure an adequate amount of calcium intake to reduce the risk of osteoporosis.

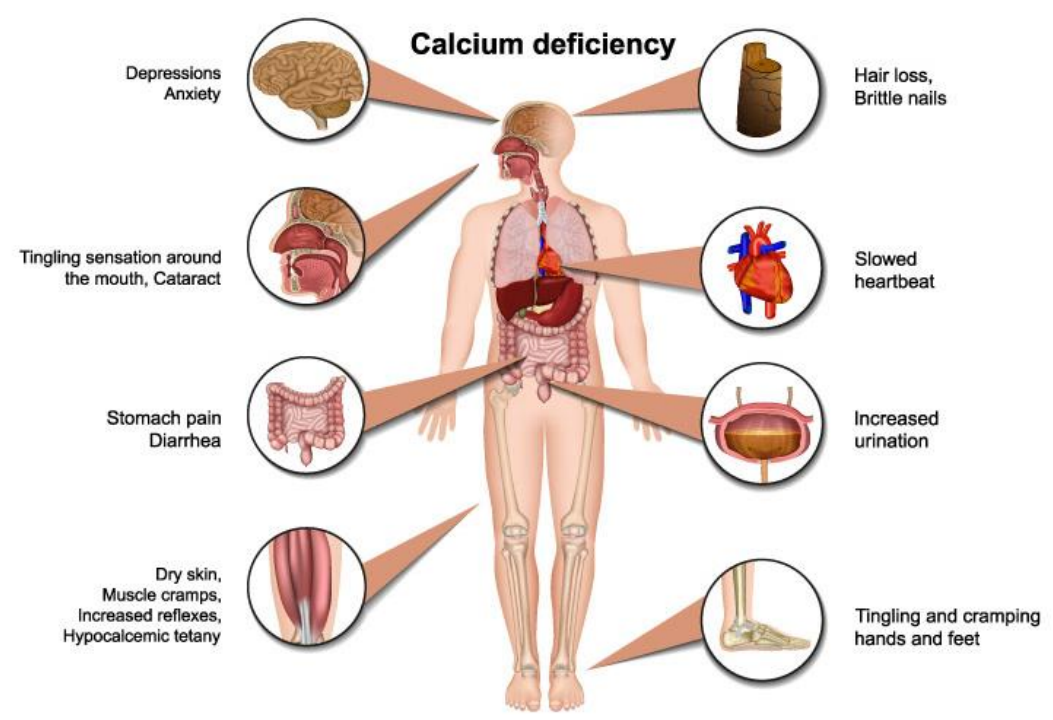

Fig.2. Calcium Deficiency - Issues (Source: https://www.yohindi.in)

\section{Types of calcium deficiency}

There are two types of calcium deficiency

Dietary calcium deficiency is a condition in which there is an inadequate calcium intake, which can lead to depleted calcium stores in the bones, thinning and weakening of the bones, and osteoporosis. 
Hypocalcemia is a low level of calcium in the blood. It can occur from taking medications, such as diuretics; medical treatments; or disease processes, such as renal failure or hypo-parathyroidism. An insufficient amount of calcium in our diet will generally not cause hypocalcemia. This is because normal amounts of calcium in the blood are so critical to many vital body functions of the nerves, muscles, brain and heart, that our body will pull calcium from the bones as needed to maintain normal blood calcium levels. This enables important processes in the body to continue. However, ongoing dietary calcium deficiency can eventually lead to thinning of the bones and osteoporosis because calcium stores in the bones are not replaced as they are used by the body. Untreated calcium deficiency can lead to serious complications, such as osteoporosis, hypertension and cardiac arrhythmias.

\section{Sign of Deficiency in Calcium}

All humans lose bone density starting between the ages of 30 and 40. Excessive bone loss affects over20 million people, mostly women who are 45 and older.

\section{Sign no. 1: Muscle Cramping}

One of the first signs of a deficiency is a nervous affliction called tetany, which is characterized by muscle cramps, numbness and tingling in the arms and legs. Muscle Cramping can be an early sign that you are developing a calcium deficiency. These types of cramps generally occur at night, especially in the legs.

\section{Sign no. 2: Dry Skin and Brittle Nails}

A common calcium deficiency sign can be seen in our skin and our nails. When our skin becomes dry and our fingernails become brittle (break easily), you could be lacking from calcium. If these symptoms are present, you may also want to check to see if our teeth are becoming yellow. The teeth and the bones can be severely affected from a lack of calcium.

\section{Sign no. 3: Increased PMS Symptoms}

A woman may begin experience more cramping or a change in her menstrual flow if she is suffering from a calcium deficiency. Adding more calcium to a diet may ease these symptoms.

\section{Sign no. 4: Bone Fractures or Breakage}

If you begin to suffer from several small bone fractures or full bone breakage, you should really evaluate the amount of calcium in our diet. This is a severe symptom of calcium deficiency.

Calcium is needed to build bones and to keep them strong. Without this calcium, our bones will become weak. As they weaken, fractures and breakage can occur i.e., osteoporosis, in which the bones become porous and fragile because calcium is withdrawn from the bones and other areas faster than it is deposited in them.

Moderate cases of calcium deficiency may lead to cramps, joint pains, heart palpitations, increased cholesterol levels, slow pulse rates, insomnia, impaired growth, excessive irritability or nerves, muscle cramps, brittle nails, eczema and numbness of the arms and or legs. A deficiency may be due to a lack of vitamin D or abnormal concentrations of hormones that regulate the availability from the bones to the blood, not to a dietary inadequacy. 
Calcium is vital for building strong bones and teeth, muscle function, release of hormones and enzymes, and assists nerves in transmitting impulses. Fructooligosaccharides may also be of benefit in helping to relieve constipation) and other gastrointestinal disorders, including problems related to irritable bowel syndrome, inflammatory bowel disease, and lactose intolerance.

Magnesium is needed for making new cells, activating B vitamins, relaxing muscles, clotting blood, energy metabolism, and is concentrated in the bones and teeth. It helps keep cells electrically stable, maintains proper blood pressure, and, with calcium, regulates energy levels and maintains normal heart function and nerve transmission. Vitamin D regulates calcium metabolism, which is important for the formation of bones and teeth. Zinc is an essential trace mineral that is necessary for the activity of 300 or more different enzymes. Special chelates ('claws' that hold the nutrient and allow for better bioavailability) enhance the delivery and utilization of the minerals.

\section{Composition}

\section{Calcium - $214 \mathrm{mg}$}

As Calcium citrate malate $-1000 \mathrm{mg}$

Fructoligosaccharides - $200 \mathrm{mg}$

Vitamin D2 - $5 \mathrm{mcg}$

Magnesium - $12.85 \mathrm{mg}$

Zinc - 10 mg

Copper - $1.0 \mathrm{mg}$

Manganese - $2.5 \mathrm{mg}$

\section{Pharmacological Action}

Calcium citrate malate (500mg) - calcium bound citrate and malic acid clinically proven as one of the most absorbable calcium form with the solubility as high as $42 \%$ compared to $22 \%$ of calcium carbonate.

Fructoligosaccharides - Prebiotic for well-functioning of intestinal ecosystem \& for healthy body. It also boosts absorption of calcium and Magnesium.

Vitamin D2 - Increases bone mass \& reduces the risk of fracture.

Magnesium - Amino acid chelated minerals

Zinc, copper and Manganese - vital support for bone metabolism.

\section{Usage}

CC Mate tablets are used in the treatment of calcium deficiency states which may occur in diseases such as hypoparathyroidism (acute and chronic); pseudohypoparathyroidism, Postmenopausal and senile osteoporosis, rickets and osteomalacia. 


\section{Contra-indications}

Absolute contra-indications are hypercalcaemia resulting for example from myeloma, bone metastases or other malignant bone disease, sarcoidosis; primary hyperparathyroidism and vitamin D overdosage.

Relative contra-indications are osteoporosis due to prolonged immobilisation, renal stones, severe hypercalciuria. Hypersensitivity to any of the tablet ingredients.

Severe renal failure.

\section{Dosage and Administration}

Adults and Elderly and children above 12 years of age: 2-3 tablets daily with meals or as directed by the health care practitioner.

Children: Not recommended for children under 12 years.

Administration: It may be taken at any time with or without food.

\section{Warnings}

Patients with mild to moderate renal failure or mild hypercalciuria should be supervised carefully including periodic checks of plasma calcium levels and urinary calcium excretion.

In patients with a history of renal stones urinary calcium excretion should be measured to exclude hypercalciuria. With long-term treatment it is advisable to monitor serum and urinary calcium levels and kidney function, and reduce or stop treatment temporarily if urinary calcium exceeds $7.5 \mathrm{mmol} / 24$ hours ( $300 \mathrm{mg} / 24$ hours).

Caution is required in patients receiving treatment for cardiovascular disease.

CC Mate tablets should also be used with caution in other patients with increased risk of hypercalcaemia e.g., patients with sarcoidosis or those suffering from malignancies.

\section{Side Effects}

The use of calcium supplements has, rarely, given rise to mild gastro-intestinal disturbances, such as constipation, flatulence, nausea, gastric pain, diarrhoea.

Following administration of vitamin D supplements occasional skin rash has been reported. Hypercalciuria, and in rare cases hypercalcaemia have been seen with long-term treatment at high dosages.

No significant side effects have yet been reported.

\section{Drug Interactions}

The risk of hypercalcaemia should be considered in patients taking thiazide diuretics since these drugs can reduce urinary calcium excretion. Hypercalcaemia must be avoided in digitalized patients.

Certain foods (e.g., those containing oxalic acid, phosphate or phytinic acid) may reduce the absorption of calcium. 
Concomitant treatment with phenytoin or barbiturates can decrease the effect of vitamin D because of metabolic activation. Concomitant use of glucocorticoids can decrease the effect of vitamin D.

The effects of digitalis and other cardiac glycosides may be accentuated with the oral administration of calcium combined with Vitamin D. Strict medical supervision is needed and, if necessary monitoring of ECG and calcium.

Calcium salts may reduce the absorption of thyroxine, bisphosphonates, sodium fluoride, quinolone or tetracycline antibiotics or iron. It is advisable to allow a minimum period of four hours before taking the calcium.

\section{Known symptoms of Overdosage and particulars of its Treatment}

The most serious consequence of acute or chronic overdose is hypercalcaemia due to vitamin D toxicity. Symptoms may include nausea, vomiting, polyuria, anorexia, weakness, apathy, thirst and constipation. Chronic overdoses can lead to vascular and organ calcification as a result of hypercalcaemia. Treatment should consist of stopping all intakes of calcium and vitamin D and rehydration.

\section{Conclusion}

A good accumulation of calcium in the bones at early stages in life is the best prevention of age-related bone loss and fractures. It is important to include adequate amounts of calcium in their daily diet. It is more efficient to take calcium in smaller doses several times a day and at night before bedtime, which also promotes a sound sleep.

When there is not enough calcium absorbed in the body, the output of estrogen decreases. As is the case with postmenopausal women, older men are often deficient in calcium. Even it also can be encourages moderate exercise. Although dairy products are the main source of calcium in the diet, other foods also contribute to overall calcium intake. Calcium is also used in muscle contraction, blood clotting, and maintenance of cell membranes. Long-term calcium deficiency can lead toosteoporosis.CC Mate tablets are effective in calcium deficiency states which may occur in diseases such as hypoparathyroidism (acute and chronic); pseudohypoparathyroidism, Postmenopausal and senile osteoporosis, rickets and osteomalacia.

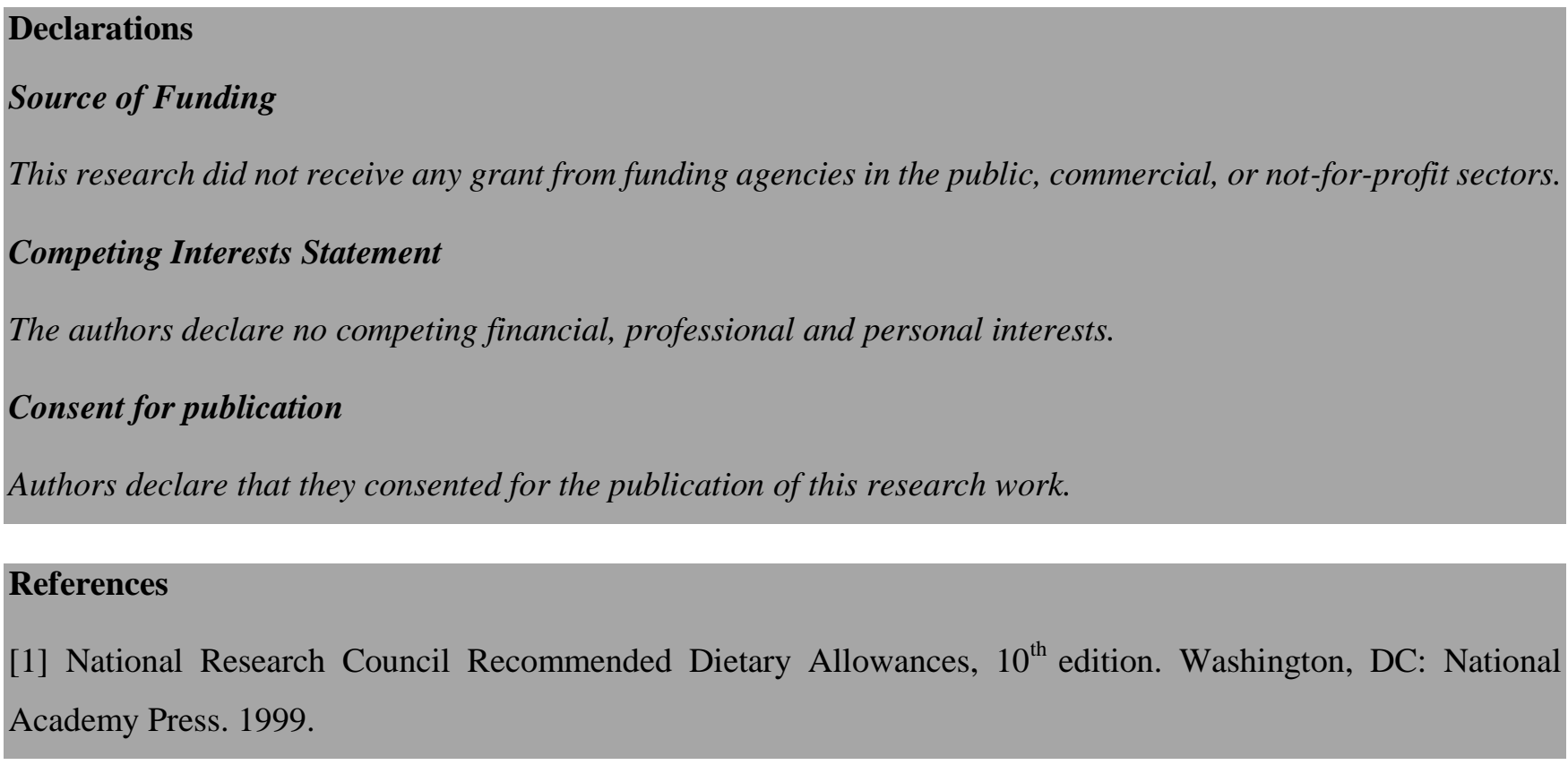


Mediterranean Journal of Basic and Applied Sciences (MJBAS)

Volume 5, Issue 4, Pages 120-127, Oct-Dec 2021

[2] Olendorf D, Jeryan C and Boyden K. The Gale Encyclopedia of Medicine. Farmington Hills, MI: Gale Research. 1999.

[3] Aaron JE, Gallagher JC, Anderson J, Stasiak L, Longton EB, Nordin BE, Nicholson M. Frequency of osteomalacia and osteoporosis in fractures of the proximal femur. Lancet. 1974 Feb 16; 1(7851): 229-33. doi: 10.1016/s0140-6736(74)92545-8. PMID: 4130245.

[4] Albright F, Bloomberg E, Drake T, Sulkowitch HW. A comparison of the effects of a.t. 10 (dihydrotachysterol) and vitamin d on calcium and phosphorus metabolism in hypoparathyroidism. J Clin Invest. 1938 May; 17(3): 317-29. doi: 10.1172/JCI100956. PMID: 16694576; PMCID: PMC424995.

[5] Consensus Development Conference (1993) Diagnosis, Prophylaxis, and Treatment of Osteoporosis. The American Journal of Medicine, 94, 646-650.

[6] Baker MR, McDonnell H, Peacock M, Nordin BE. Plasma 25-hydroxy vitamin D concentrations in patients with fractures of the femoral neck. Br Med J. 1979; 1 (6163): 589. doi: 10.1136/bmj.1.6163.589.

[7] Bouillon RA, Auwerx JH, Lissens WD, Pelemans WK. Vitamin D status in the elderly: seasonal substrate deficiency causes 1, 25-dihydroxycholecalciferol deficiency. Am J Clin Nutr. 1987 Apr; 45(4): 755-63. doi: 10.1093/ajcn/45.4.755. PMID: 3494392.

[8] Boyce WJ, Vessey MP. Rising incidence of fracture of the proximal femur. Lancet. 1985 Jan 19; 1(8421): 150-1. doi: 10.1016/s0140-6736(85)91915-4. PMID: 2857223.

[9] Neil A. Breslau, James L. Mcguire, Joseph E. Zerwekh, Charles Y. C. Pak, The Role of Dietary Sodium on Renal Excretion and Intestinal Absorption of Calcium and on Vitamin D Metabolism, The Journal of Clinical Endocrinology \& Metabolism, Volume 55, Issue 2, 1 August 1982, Pages 369-373, https://doi.org/10.1210/ jcem-55-2-369.

[10] Bronner F. Intestinal calcium absorption: mechanisms and applications. J Nutr. 1987 Aug; 117(8): 1347-52. doi: 10.1093/jn/117.8.1347. PMID: 3305814.

[11] Carlsson, A.; Lindquist, B. Comparison of intestinal and skeletal effects of vitamin D in relation to dosage. Acta Physiol. Scand. 1955, 35, 53-55.

[12] Chapuy MC, Arlot ME, Duboeuf F, Brun J, Crouzet B, Arnaud S, Delmas PD, Meunier PJ. Vitamin D3 and calcium to prevent hip fractures in elderly women. N Engl J Med. 1992 Dec 3; 327(23): 1637-42. doi: 10.1056/NEJM199212033272305. PMID: 1331788.

[13] Chapuy MC, Durr F, Chapuy P. Age-related changes in parathyroid hormone and 25 hydroxycholecalciferol levels. J Gerontol. 1983 Jan; 38(1): 19-22. doi: 10.1093/geronj/38.1.19. PMID: 6600237.

[14] Christiansen C, Riis BJ. 17 Beta-estradiol and continuous norethisterone: a unique treatment for established osteoporosis in elderly women. J Clin Endocrinol Metab. 1990 Oct; 71(4): 836-41. doi: 10.1210/jcem-71-4-836. PMID: 2205624.

[15] Civitelli R, Agnusdei D, Nardi P, Zacchei F, Avioli LV, Gennari C. Effects of one-year treatment with estrogens on bone mass, intestinal calcium absorption, and 25-hydroxyvitamin D-1 alpha-hydroxylase reserve in postmenopausal osteoporosis. Calcif Tissue Int. 1988 Feb; 42(2): 77-86. doi: 10.1007/BF02556338. PMID: 3127028. 